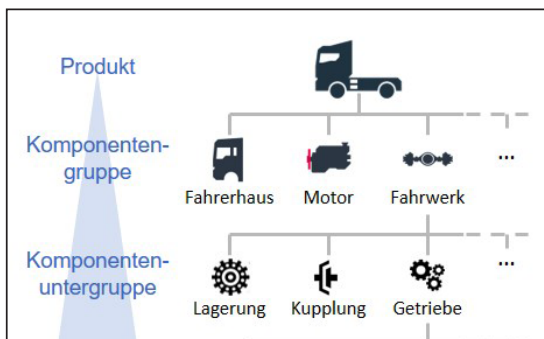

\section{Sebastian Beckschulte et al.: Manuelle Fehleraufnahme bei Mass Customization}

In der Nutzfahrzeugindustrie sorgt Mass Customization für eine Variantenvielfalt, die der Losgröße 1 entspricht. Das hat zur Folge, dass Montageprozesse einen geringen Automatisierungsgrad haben und die Fehleranfälligkeit steigt. Doch wie werden Miitarbeitende dazu befähigt, identifizierte Fehler schnellstmöglich und strukturiert zu erfassen? Hierzu wurde ein Prozessmodell entwickelt, das diese Anforderungen anwendungsgerecht adressiert.

\title{
Dominic Behle:
}

\section{Vorgehensweise zur Gestaltung eines Produktionssteuerungssystems}

Bei der Optimierung der Produktionssteuerung wird viel Arbeit in die richtigen Algorithmen investiert. Dieser Beitrag zeigt, dass vor der Optimierung eines Algorithmus strategische Fragen beantwortet werden müssen, aus denen sich das System der Produktionsplanung ergibt. Diese strategischen Dimensionen werden dargelegt und mögliche Systeme diskutiert.

\section{Mark Sanders et al.: \\ On-Machine Measurements im Internet of Production}

Bei On-Machine Measurements werden Bauteile direkt mit dem Messtastersystem der Maschine gemessen. So wird im Vergleich zur Messung per KMG der Aufwand im Vergleich zur dimensionellen Bauteilprüfung und einer anschließenden Korrektur fehlerhafter Bauteile erheblich reduziert. In diesem Beitrag wird eine Dateninfrastruktur vorgestellt, welche die aus der Maschine stammenden Daten annotiert speichert. Mithilfe der gesammelten Daten kann die Maßhaltigkeit des Bauteils automatisch erkannt werden.

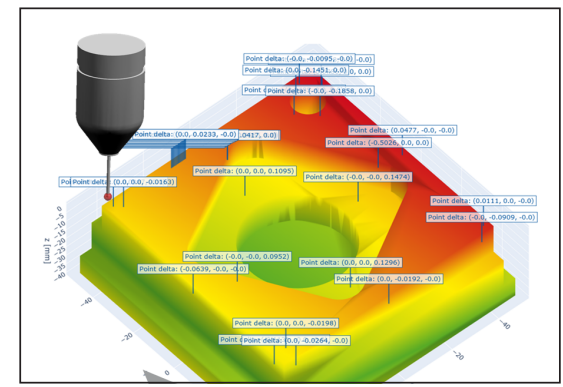

ISSN 0947-0085 · e-ISSN 2511-0896

Die ZWF ist Organ des VDI-Kompetenzfeldes Informationstechnik (VDI-KfIT).

Alle Informationen bezüglich Autorenrichtlinien, Abonnements, Open Access Publikationen, zurückliegender Jahrgänge und Bestellungen finden Sie online unter: http://www.degruyter.com/zwf

\section{HERAUSGEBER}

Prof. Dr-Ing. Prof. e.h. Wilhelm Bauer, Fraunhofer-Institut für Arbeitswirtschaft und Organisation IAO, Nobelstraße 12, 70569 Stuttgart, Germany

Tel.: +4971197020 90, e-mail: wilhelm.bauer@iao.fraunhofer.de

Prof. Dr-Ing. Wolfram Volk, Technische Universität München, Lehrstuhl für Umformtechnik und GieBereiwesen (utg), Walther-Meißner-Straße 5, 85747 Garching, Germany

Tel.: +498928913791, e-mail: wolfram.volk@utg.de

Prof. Dr.-Ing. Michael F. Zäh, Technische Universität München, Institut für Werkzeugmaschinen und Betriebswissenschaften (iwb), Boltzmannstraße 15, 85748 Garching, Germany

Tel.: +49 89289155 02, e-mail: michael.zaeh@iwb.tum.de

\section{ADVISORY BOARD}

Prof. Dr.-Ing. Jan C. Aurich. TU Kaiserslautern

Prof. Dr.-Ing. Wilhelm Bauer, Universität Stuttgart

Prof. Dr.-Ing. Dirk Biermann, TU Dortmund

Prof. Dr.-Ing. Christian Brecher, RWTH Aachen

Prof. Dr.-Ing. Martin Eigner, TU Kaiserslautern

Prof. Dr-Ing Jürgen Fleischer, Karlsruher Institut für Technologie

Prof. Dr-Ing Jürgen Gausemeier, Universität Paderborn
Prof. Dr.-Ing. Dipl.-Wirtsch.-Ing. Peter Groche, TU Darmstadt

Prof. Dr.-Ing. Steffen Ihlenfeldt, TU Dresden

Prof. Dr.-Ing. Jörg Krüger, TU Berlin

Prof. Dr.-Ing. Gisela Lanza, Karlsruher Institut für Technologie Prof. Dr.-Ing. habil. Marion Merklein, FAU Erlangen-Nürnberg Prof. Dr.-Ing. habil. Peter Nyhuis, Leibniz Universität Hannover Prof. Dr.-Ing. Dipl.-Wirt. Ing. Günther Schuh, RWTH Aachen Prof. Dr.-Ing. Dr.-Ing. E.h. A. Erman Tekkaya, TU Dortmund Prof. Dr.-Ing. Wolfram Volk, TU München

Prof. Dr.-Ing. Michael F. Zäh, TU München

\section{VERLAG}

Walter de Gruyter GmbH, Berlin/Boston, Genthiner Straße 13, 10785 Berlin, Germany REDAKTION

Dipl.-Ing. Yetvart Ficiciyan (verantwortlich), Huberweg 14 E, 13599 Berlin, Germany Tel.: +49 30221905 53, Mobil: +49 17360407 41, e-mail: zwf@mediatech-berlin.de

\section{ANZEIGENLEITUNG}

Markus Kügel, De Gruyter, Rosenheimer Str. 143, 81671 München, Germany

Tel.: +49 8976 902-424, e-mail: markus.kuegel@degruyter.com

(C) 2021 Walter de Gruyter GmbH, Berlin/Boston, Germany

\section{SATZ \& DRUCK}

druckhaus köthen GmbH \& Co. KG, Friedrichstr. 11/12, 06366 Köthen, Germany 